\title{
GÊNIO: UM GATEWAY PARA AUTOMAÇÃO PREDIAL COM INTERFACE DE VOZ
}

\author{
Gustavo Borba*, Anolan Milanés*, Amanda Borba ${ }^{\dagger}$ \\ *CEFET-MG, Nova Gameleira, Belo Horizonte, MG, Brasil \\ $\dagger U F M G$, Belo Horizonte, $M G$, Brasil
}

Emails: borba@cefetmg.br, anolan@cefetmg.br, afmb@ufmg.br

\begin{abstract}
Interest in voice control solutions has grown because of its potential for accessibility, ease of use and intuitiveness. For marketing reasons, technologies in this field are mainly focused on english speakers and resources for Brazilian Portuguese are still limited. Furthermore, many of it's services are dominated by large companies, whose solutions are highly priced, making it unaffordable for most of the brazilian population. This work aims to construct a low cost intelligent $h u b$ for home automation, entirely open source, interacting with the user through voice commands in Brazilian Portuguese. The prototype was built with Snowboy library for keyword detection, CMUSphinx tool for speech recognition and Home Assistant toolkit for devices integration. It was verified that the prototype meets the offline processing and command comprehension in the Portuguese language requirements, and has lower cost than consolidated platforms in the market.
\end{abstract}

Keywords_ Wireless Network Sensors, Smart Hubs, Home Automation, Speech Recognition

\begin{abstract}
Resumo- Soluções de controle de dispositivos por voz têm ganhado espaço por apresentarem uma tecnologia acessível, amigável e intuitiva. Por questões mercadológicas, as tecnologias nesse âmbito geralmente se apresentam na língua inglesa ou com recursos limitados para o português brasileiro. Ademais, muitos dos serviços utilizados são dominados por grandes empresas, cujas soluções têm um preço inacessível para a maioria da população no Brasil. Este trabalho expõe a construção de um hub inteligente para automação doméstica de baixo custo e inteiramente em código aberto, que interage com o usuário por comandos de voz na língua portuguesa. Para a construção do protótipo são utilizadas as bibliotecas Snowboy para detecção de palavra-chave, CMUSphinx para reconhecimento de fala e o toolkit Home Assistant para integração entre dispositivos inteligentes. Verifica-se que o protótipo atende aos requisitos de processamento offline e compreensão de comandos na língua portuguesa, e apresenta custo menor que plataformas consolidadas no mercado.
\end{abstract}

Palavras-chave — Redes de Sensores Sem Fio, Hub inteligente, Automação Predial, Reconhecimento de Fala

\section{Introdução}

O controle de dispositivos domésticos, como medição e manutenção da qualidade do ar, iluminação, segurança, consumo eficiente de energia, entre outros é conhecido por Automação Predial ou Domótica (Trappeniers, 2013). A área tem sido auxiliada pela recente expansão de mercado da Internet das Coisas: objetos inteligentes (equipados com sensores, atuadores e processadores), serviços e aplicações interconectados por meio da Internet (Sethi and Sarangi, 2017). Um dos domínios mais reconhecidos da automação predial é a acessibilidade com foco na crescente população idosa que, segundo um estudo publicado pela Organização Mundial de Saúde (Organization, 2015), chegará a 2 bilhões de pessoas até 2050 . Soluções de controle de dispositivos por voz têm ganhado espaço por constituírem uma tecnologia amigável e intuitiva. Portet (2011) alega que tais sistemas, aliados à Internet das Coisas (IoT), são mais aceitos do que soluções consideradas intrusivas (diferentes, por exemplo, de sensores fisiológicos que devem ser utilizados 24 horas por dia).

Segundo Mahmoud (2014), a estratégia explorada atualmente para disponibilizar soluções de controle por voz é a utilização de gateways como agentes supervisórios de objetos conectados à rede. Isso significa aproveitar-se da maior capacidade de processamento com relação aos demais dispositivos da rede que gateways dispõe e sua interoperabilidade entre redes para fazer o papel de interface com o usuário para diversos serviços, exibir múltiplas interfaces de comunicação e atuar como controle remoto para esses dispositivos.

Infelizmente, as alternativas comerciais disponíveis no país dependem de conexão à rede de alta velocidade, exigindo altos custos de projeto e manutenção, e têm baixo suporte para a língua portuguesa. Um dos motivos para isso é a necessidade de grandes bases de dados para a interpretação de voz de forma generalista. Um dos motivos para a diminuta quantidade de plataformas de código aberto e baixo custo de controle por voz em português é a falta de corpus linguístico. Em um estudo comparativo, Moore (2003) indica a necessidade de uma "quantidade fantástica" de dados para atingir um reconhecimento preciso da fala humana, chegando a ser de cem a mil vezes maior do que a quantidade necessária para uma criança aprender a falar.

Além do problema da quantidade de dados necessária, ainda há a preocupação com a privacidade do usuário. Ao consumir um serviço hospedado na nuvem, um usuário envia sua voz gravada para os servidores das empresas que hospedam o serviço. Como esse dado será utilizado, além da função-fim prestada de identificação de fala, ainda é um ponto forte de desconfiança (Balta-Ozkan et al., 2014). Uma alternativa para tal preocupa- 
ção seria a confecção de uma ferramenta que funcione offline, ou que garanta que nenhum dado sensível do usuário será enviado para servidores.

No decorrer deste trabalho não foram encontradas soluções de código aberto que incorporassem todas as funções necessárias para um hub inteligente para a língua portuguesa sem que se utilizasse um serviço de terceiros hospedado na nuvem. Estas funções são: reconhecimento de voz, processamento de linguagem natural, sintetização de voz e comunicação entre dispositivos. Nesse artigo é proposto o protótipo de um smart hub com interface amigável baseada em comandos de voz em português brasileiro para o controle de dispositivos de sistemas de automação predial. Utilizando apenas tecnologias open source, o dispositivo é capaz de supervisionar os dispositivos inteligentes conectados via rede sem a necessidade de realizar o processamento de voz em um servidor externo.

\section{Projeto e Arquitetura}

\subsection{Arquitetura de Software}

Como mostra a Figura 1, o projeto abarca ao menos dois dispositivos comunicantes via $\mathrm{Wi}-\mathrm{Fi}$, sendo o primeiro o hub inteligente, e o segundo um dispositivo inteligente acionado por esse $h u b$. O protótipo do hub inteligente foi desenhado para se situar nas três camadas do meio da arquitetura de cinco camadas proposta por (Sethi and Sarangi, 2017), a saber, aplicação, processamento e transporte. Atua na camada de aplicação realizando a transcrição de voz para texto e viceversa, servindo como interface para o usuário. $\mathrm{Na}$ camada de processamento são identificadas as intenções desejadas (atuações dos dispositivos: "ligar", "abrir", "tocar uma música", etc) e os objetos envolvidos na ação que o usuário propõe. Nessa camada é realizado um parse e identificado o dispositivo a ser acionado segundo uma tabela de nomes. Por fim, na camada de transporte, o hub envia o comando para o atuador identificado. $\mathrm{O}$ dispositivo inteligente, por sua vez, atua apenas na camada de percepção, atuando sobre o dispositivo (seja uma lâmpada, um eletrodoméstico ou uma tranca eletrônica).

O projeto utiliza a plataforma Home Assistant (HA) como motor principal, bem como centralizador da troca de mensagens entre os componentes escritos (HomeAssistant, 2018). O processo de criação de um assistente virtual pode ser separado em 5 fases como descrito por Hansen (2018) e ilustrado pela Figura 2. Primeiramente, um serviço de deteç̧ão de palavra-chave que roda em background reconhece a palavra-chave "Gênio". Um segundo serviço, responsável pela função de reconhecimento de fala, transcreve o áudio dito para texto. O texto identificado é então enviado para um terceiro serviço, que indexa cada texto
Figura 1: Categorização do projeto na arquitetura de 5 camadas

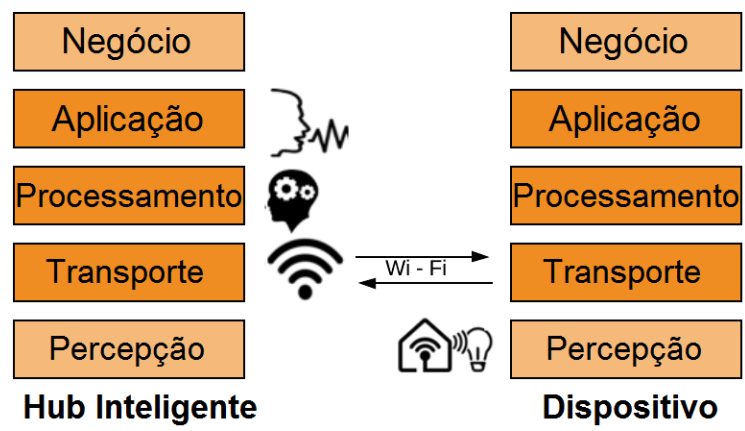

Fonte: Adaptado de Sethi \& Sarangi (2017)

com seu dispositivo e a ação desejada. Em um quarto passo, a aplicação HA é responsável por enviar a mensagem para o dispositivo, que executará a ação. Ao mesmo tempo, um último serviço de Text-To-Speech (TTS) fará a transcrição de uma mensagem de resposta ao usuário, indicando se a ação foi corretamente interpretada ou não.

Figura 2: Processo de atuação da plataforma Gênio

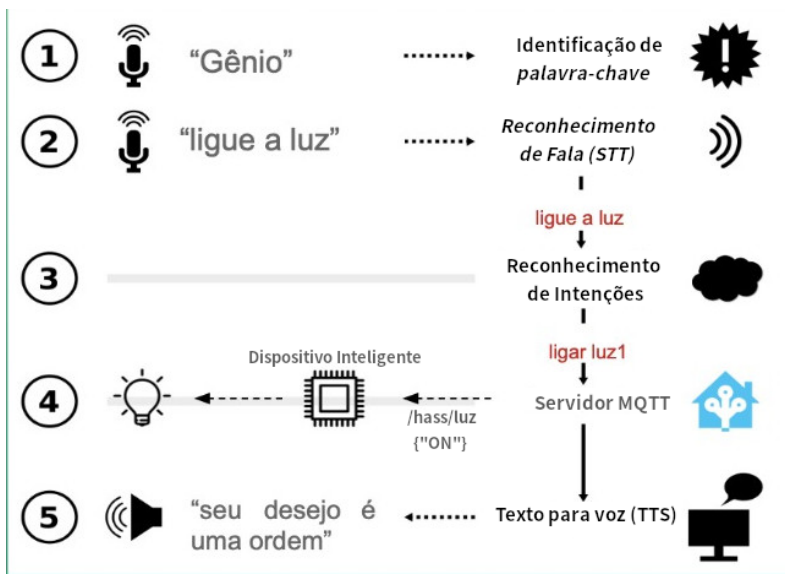

Fonte: adaptado de Hansen (2018)

Como a plataforma HA é desenvolvida em Python, o código dos componentes citados foi escrito nessa linguagem, para manter a padronização do projeto. A biblioteca Snowboy ${ }^{1}$ foi escolhida como serviço de reconhecimento de palavra-chave, por ser uma ferramenta de código aberto facilmente treinável e configurável. O serviço de Identificação de voz foi desenvolvido utilizando a biblioteca SpeechRecognition, sob o motor CMUSphinx (CMUSphinx, 2018). Da mesma linguagem, também foi utilizada a biblioteca pyttsx para transcrição de texto para voz.

\subsection{Comunicação entre os componentes}

A solução pressupõe que ambos os dispositivos estejam conectados na mesma rede Wi-Fi, e a comu-

\footnotetext{
${ }^{1}$ Disponível em https://snowboy.kitt.ai/. Acesso em 28 de Setembro de 2018
} 
Figura 3: Fluxo de informação entre os componentes desenvolvidos

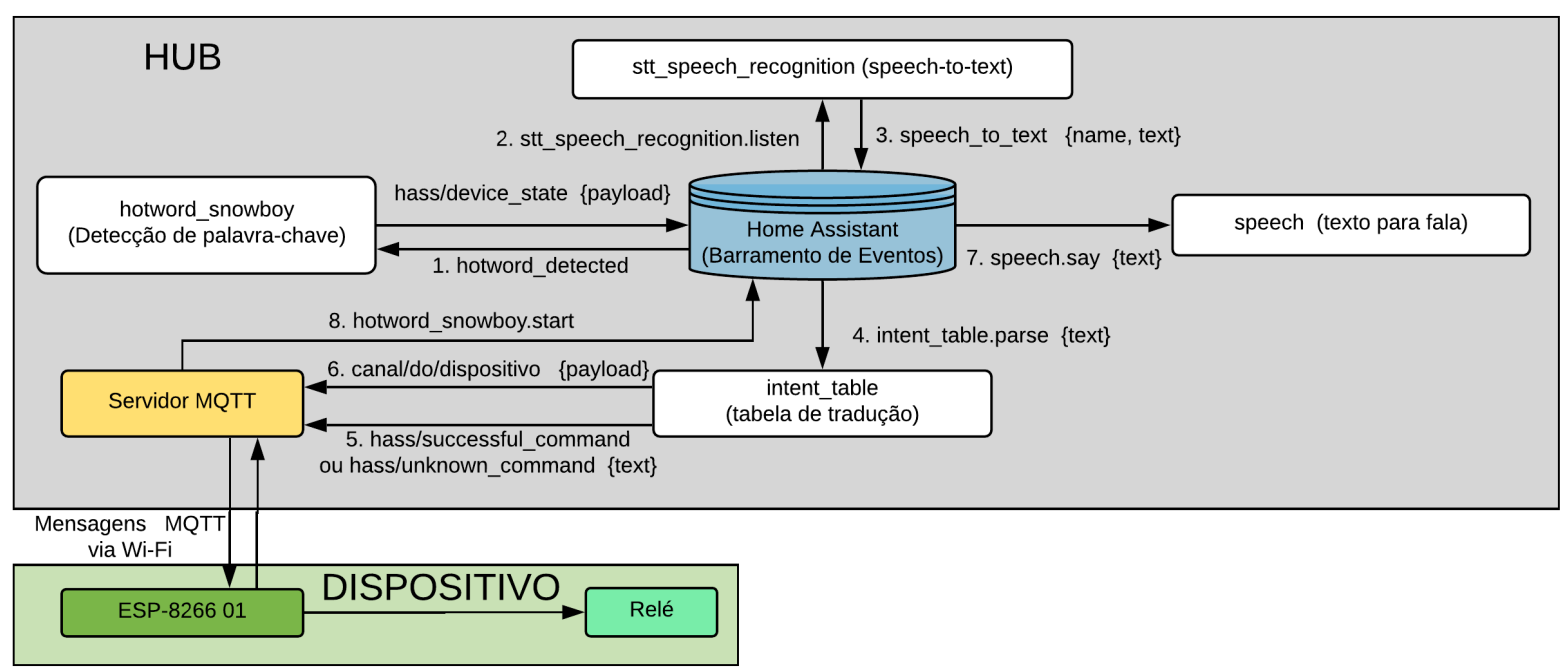

Fonte: elaborado pelo autor

nicação entre $h u b$ e dispositivo é feita utilizando o protocolo MQTT (OASIS, 2014). Este é um protocolo comunicação no estilo publicar-assinar (publish-subscribe), onde publicadores divulgam informações através de eventos para um serviço de evento intermediário e consumidores são assinantes de eventos específicos deste serviço. Esse paradigma de comunicação permite que não haja acoplamento direto entre componentes comunicantes. Segundo Coulouris (2005), esse desacoplamento pode ser diferenciado em espacial e temporal:

1. Desacoplamento espacial - Ocorre quando o remetente não sabe quem são os destinatários da mensagem que está enviando.

2. Desacoplamento temporal - Ocorre quando o sistema do remetente e destinatário não precisam estar conectadas ao mesmo tempo.

O protocolo MQTT utiliza um broker central para a comunicação entre os dispositivos. A princípio, esse broker central pode ser qualquer servidor situado na mesma rede Wi-Fi do protótipo e dos dispositivos. Para fins de simplificação do projeto, um servidor MQTT foi instalado no mesmo dispositivo que hospeda a aplicação proposta.

Os componentes de software responsáveis pelas funções descritas na seção anterior se comunicam com o motor HA de forma muito similar ao protocolo MQTT. O motor implementa seu próprio mecanismo, chamado barramento de eventos. Este se diferencia do protocolo MQTT apenas pelo payload (o formato da transmissão de dados, sem as informações utilizadas para facilitar a entrega, como cabeçalhos e metadados). Dessa forma, enquanto para acionar um dispositivo a mensagem a ser enviada para o servidor MQTT seria "ON", no canal "hass/sala/luz1/set", a mensagem para indicar a detecção da palavra-chave teria o formato "hotword: 'Gênio'" no canal hotword_detected do Barramento de Eventos.

A Figura 3 exemplifica as mensagens trocadas entre os componentes. O hub inteligente, responsável pelo processamento de voz e identificação das intenções do usuário, é representado pelo painel cinza. A base de dados em azul representa o barramento de eventos do motor HA, que gerencia as comunicações entre os processos. Os componentes de software desenvolvidos para solucionar cada uma das etapas descritas na Figura 2 estão representados na cor branca, e se comunicam via troca de mensagens pelo barramento de eventos supracitado. O servidor MQTT é representado na cor amarela pois, apesar de ser unidade constituinte do protótipo desenvolvido, não precisa ser necessariamente interno ao hub inteligente. Por fim, o dispositivo inteligente é representado na cor verde. É importante frisar que todas as formas de comunicação entre módulos e dispositivos é de natureza assíncrona, garantindo mais liberdade entre componentes de software, mas ao mesmo tempo adicionando complexidade ao sistema.

\subsection{Componentes de Hardware}

O gateway com interface de voz foi instalado em um microcomputador de placa única Raspberry Pi 3B. Essa placa contém um processador Quad Core de 64 bits, GPU integrada, saídas de vídeo HDMI e sinal de áudio. Disponibiliza ainda uma entrada Ethernet e quatro entradas USB, mas não apresenta entradas de áudio. Foi utilizado um microfone USB, embora configurações adicionais se tornaram necessárias para que as bibliotecas de captura de voz funcionem corretamente.

O microcontrolador escolhido para os sensores e atuadores digitais foi o ESP8266-01. O ESP8266 é um microcontrolador produzido pela empresa Espressif Systems. Seus grandes diferenciais são 
possuir um sistema de comunicação Wi-Fi próprio e seu baixo custo . Esse microcontrolador pode rodar suas próprias aplicações se comunicando via Wi-Fi e também pode servir de adaptador Wi-Fi para outro microcontrolador, como um Arduino ou PIC. O programa executado no microcontrolador assume um conhecimento por parte do projetista dos parâmetros da rede e do dispositivo, sejam eles o nome e a senha da rede, o endereço IP do servidor MQTT e os canais MQTT que serão assinados e que as mensagens serão publicadas.

Figura 4: Esquema do dispositivo inteligente

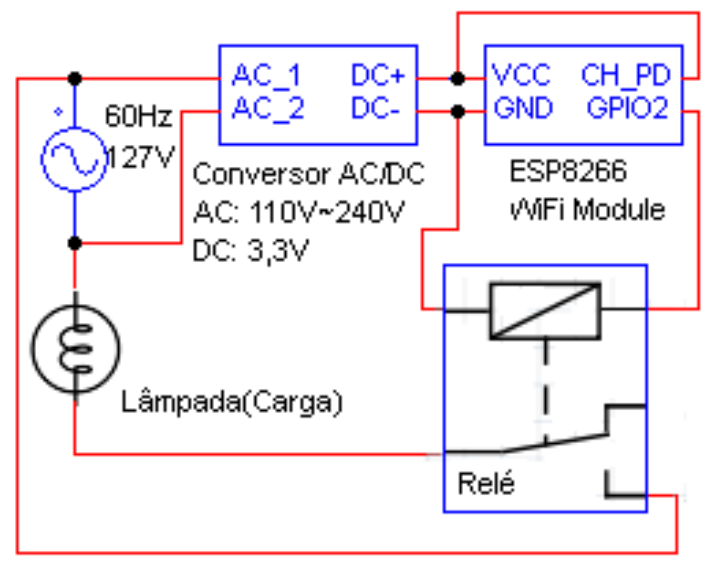

Fonte: elaborado pelo autor

A modelagem do dispositivo inteligente a ser acionado pelo $h u b$ levou em consideração a sua capacidade de ser autocontido. O dispositivo implementado pode ser contido em uma caixa de passagem e apresenta apenas três conexões externas (entradas da rede analógica e saída do comando para a lâmpada). Dessa forma, o dispositivo é passível de ser instalado em qualquer rede doméstica. Foi utilizado um módulo com relé de estado sólido para a ativação do componente a ser controlado e uma lâmpada para representar esse dispositivo (que não necessariamente precisa ser um dispositivo luminoso, pode ser uma tranca, uma luz, um eletrodoméstico, etc), um retificador chaveado de 127 VAC para +5VDC (aproveitada de um carregador de celular) e foi produzido um circuito redutor de tensão com dois diodos e um capacitor.

Na Figura 4, o retificador chaveado retira sua força da rede elétrica, que também alimenta a carga controlada pelo dispositivo inteligente, representado por uma lâmpada. Os dois diodos e o capacitor cumprem a função de retificação de tensão, alimentando tanto o ESP8266, microcontrolador e responsável pela conexão Wi-Fi do dispositivo, quanto o relé que ativará a carga.

\subsection{Reconhecimento de Voz}

A biblioteca Python SpeechRecognition permite a utilização de diferentes motores de reconhecimento de fala, a parte mais desafiadora ao se projetar um assistente doméstico. Para o processa- mento ser realizado no próprio dispositivo, foi utilizando o motor CMUSphinx, cujo núcleo é composto de três componentes: um dicionário fonético, um modelo acústico e um modelo de linguagem. Para a geração dos modelos acústico e de linguagem, é necessário treinar um corpus linguístico. O Corpus de voz e texto da Constituição Federal utilizado foi disponibilizado pelo grupo $F a$ laBrasil ${ }^{2}$, foi possível criar um modelo acústico e de linguagem pelo aplicativo sphinxtrain, parte integrante do motor CMUSphinx. Esse passo foi realizado seguindo o tutorial para treinamento de modelos acústicos disponibilizado no site do projeto CMUSphinx (CMUSphinx, 2018). Com uma precisão mais modesta, apenas cerca de metade das frases ditas foram devidamente reconhecidas. Para melhorar esse resultado, o dicionário fonético foi reduzido de forma manual.

Apenas o uso dos artefatos gerados pelo corpus da Constituição Federal não obteve melhoras significativas na interpretação da fala. Foi necessário treinar o modelo de linguagem utilizando apenas as palavras do dicionário reduzido. Para aumentar a precisão da interpretação dos comandos também foi utilizada uma gramática em formato JSGF (W3C, 2018) de forma conjunta ao modelo de linguagem.

\section{Testes e Resultados}

Para avaliar a qualidade do protótipo, foram realizados dois testes. Estes testes tiveram a finalidade de verificar a presença e qualidade de todas as funcionalidades propostas para o sistema

O primeiro teste diz respeito apenas ao dispositivo inteligente projetado. São enviadas mensagens MQTT no canal correspondente ao dispositivo, verificando se o dispositivo funciona de forma adequada à proposta.

O segundo teste planejado teve como foco o $h u b$ inteligente e, mais especificamente, avaliar a capacidade do protótipo de capturar e interpretar corretamente os comandos falados. A lista de comandos foi pré-definida, contendo uma amostra das múltiplas possibilidades de comandos de voz a serem interpretados. O sistema registrou os comandos interpretados em um arquivo de log e sua efetividade foi avaliada pela da taxa de comandos corretamente interpretados diante da quantidade de casos de teste. Foi medido também, para cada comando dito, o tempo de acionamento do dispositivo, em caso de sucesso. A métrica para avaliação desse teste foi a média de tempo para acionar os dispositivos.

Num primeiro experimento, foram enviadas diferentes mensagens ao canal MQTT "hass/quarto/luz1/set": para ligar o dispositivo inteligente, a mensagem "ON". Para desligá-lo,

\footnotetext{
${ }^{2}$ Disponível em http://labvis.ufpa.br/falabrasil/downloads/. Acesso em 05 de Setembro de 2018
} 
Tabela 1: Temporização amostrada relativa aos comandos intepretados corretamente (em segundos)

\begin{tabular}{lcccc}
\hline Comando falado & $\begin{array}{c}\text { Tempo de } \\
\text { reação }\end{array}$ & $\begin{array}{c}\text { Gravação do } \\
\text { Comando }\end{array}$ & $\begin{array}{c}\text { Reconhecimento } \\
\text { do comando }\end{array}$ & $\begin{array}{c}\text { Atuação após } \\
\text { detecção de fala }\end{array}$ \\
\hline ligar luz sala & 02 & 07 & 05 & 14 \\
desligar luz sala & 01 & 06 & 05 & 12 \\
ligar luz quarto & 01 & 05 & 05 & 11 \\
diminua o volume & 01 & 03 & 05 & 09 \\
aumente o volume & 01 & 03 & 05 & 09 \\
desligar luz & 01 & 07 & 05 & 13 \\
ligar luz cozinha & 01 & 07 & 04 & 13 \\
abra porta sala & 01 & 04 & 05 & 10 \\
desligar luz cozinha & 01 & 05 & 05 & 11 \\
ligar luz cozinha & 02 & 05 & 05 & 11 \\
\hline média & 01 & 05 & 05 & \\
\hline
\end{tabular}

"OFF". Para um comando de pulso, simbolizando uma tranca eletrônica, a mensagem "PULSE"foi enviada. Por fim, para solicitar a situação do dispositivo, a mensagem "ask_state"foi utilizada. Esse teste obteve sucesso em todos os cenários, verificando a capacidade do dispositivo responder aos comandos de forma praticamente instantânea. O computador utilizado para a programação da placa e também como servidor MQTT foi um notebook modelo Dell Vostro 5480.

O segundo experimento teve como objetivo medir o tempo de resposta e a acurácia do $h u b$ inteligente, no que diz respeito à sua capacidade de interpretar comandos de voz. Foram selecionadas 20 frases distintas, livremente escolhidas dentre as várias possibilidades disponibilizadas pela gramática. Essas frases foram faladas ao protótipo para acionamento dos dispositivos e a resposta do $h u b$ inteligente foi analisada e temporizada. Com o dispositivo montado como indicado na Figura 5, em um ambiente silencioso (os testes ocorreram durante madrugadas, em zona residencial de alta densidade), os comandos foram ditos a cerca de um metro de distância do dispositivo, em tom de voz normal, sempre pela mesma pessoa.

Figura 5: Protótipo do hub inteligente montado com microfone e alto-falante

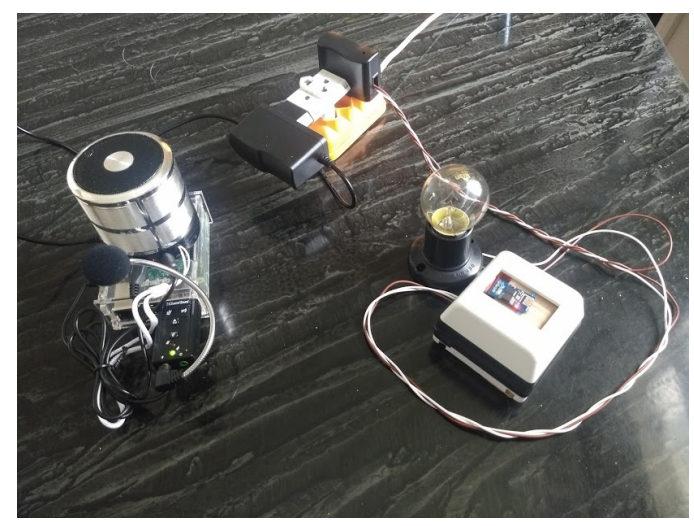

Fonte: elaborado pelo autor

Cada componente escrito tem uma tarefa crítica, seja detectar a palavra-chave, identificar voz, traduzir um comando para voz, etc. Todos os componentes implementados registraram em log o horário de início e término dessa atividade. Também foi gravado em log o comando identificado, ou caso não tenha sido identificado algum comando, a falha.

A palavra-chave foi detectada em $95 \%$ das vezes em que foi intencionalmente falada e nenhum falso-positivo foi identificado. Em testes posteriores, em um ambiente ligeiramente mais barulhento, falsos positivos ocorrem quando há grandes fontes de som (como música alta tocando ou pessoas gritando indistintamente). Observou-se que o tempo gasto do momento do início da gravação do comando de voz até a atuação se manteve constante em 5 segundos (precisamente o tempo definido para compreender o comando). De igual forma, o tempo de atualização do estado do dispositivo na ferramenta também se manteve constante em 1 segundo.

Como exibe a Tabela 1, os comandos interpretados levaram, em média, 11 segundos do momento da detecção da palavra-chave até a atuação do dispositivo. Comandos não reconhecidos tiveram seu tempo reduzido a 8 segundos. Entretanto deve-se considerar o tempo em que o usuário fala o comando (cerca de 5 segundos). Dessa forma, a atuação leva, em média de 5 a 6 segundos para estar concluída, estando dentro do limite previamente estabelecido de 5 segundos. É possível verificar, na Tabela 2, que para os comandos não reconhecidos houve um menor tempo de gravação. Isso ocorreu devido à natureza assíncrona da troca de mensagens entre os componentes: o feedback para o usuário, indicando que ele pode iniciar o comando, ocorreu durante a captação do próprio comando. Assim, o áudio captado foi justamente a mensagem de feedback dada pelo hub inteligente.

Uma análise qualitativa da interpretação dos comandos levou em conta a velocidade e naturalidade ao se falar os comandos. Ao se dizer apenas as palavras dos comandos (sem incluir os artigos e preposições naturais da fala), os comandos eram 
Tabela 2: Temporização amostrada relativa aos comandos não identificados (em segundos)

\begin{tabular}{lcccc}
\hline Comando falado & $\begin{array}{c}\text { Tempo de } \\
\text { reação }\end{array}$ & $\begin{array}{c}\text { Gravação do } \\
\text { comando }\end{array}$ & $\begin{array}{c}\text { Reconhecimento } \\
\text { do comando }\end{array}$ & $\begin{array}{c}\text { Atuação após } \\
\text { detecção de fala }\end{array}$ \\
\hline ligar luz cozinha & 01 & 02 & 05 & 08 \\
apagar luz cozinha & 01 & 03 & 05 & 09 \\
luz sala ligada? & 01 & 02 & 05 & 08 \\
luz sala ligada? & 01 & 02 & 04 & 07 \\
luz quarto ligada? & 01 & 04 & 04 & 09 \\
\hline média & 01 & 03 & 05 & 08 \\
\hline
\end{tabular}

percebidos com maior dificuldade. Ao utilizar as estruturas completas da frase, como por exemplo "desligar A luz Do quarto", adicionando maior fluidez na fala, o comando era melhor compreendido pela ferramenta. Dessa análise é possível supor que o modelo estatístico de reconhecimento de fala encontre maior verossimilhança nos comandos falados que tenham palavras auxiliares, uma vez que seu modelo é treinado a partir de frases completas advindas de livros.

\section{Conclusões e Trabalhos Futuros}

Apesar da necessidade desses pequenos ajustes, o protótipo se mostra como um bom produto mínimo viável (MVP). A ferramenta desenvolvida se mostra robusta e expansível, apesar dos dispositivos produzidos ainda necessitarem da intervenção do programador para ser instalada, por necessitar de conhecimentos da rede envolvida. A característica de maior apelo comercial do protótipo é que o reconhecimento de comandos roda localmente no dispositivo, sem depender de qualquer serviço externo. Isso garante ao usuário maior confidencialidade, já que suas gravações de voz não trafegarão por lugar algum na Internet.

Para melhorar a precisão da identificação dos comandos é recomendada a possibilidade de substituição do microfone utilizado por um mais robusto. Estudos adicionais se fazem necessários para eliminar ruídos da rede elétrica que podem não ser filtrados pela fonte de energia do Raspberry PI, computador de placa única utilizado no protótipo. Também é recomendada a utilização de Processamento de Linguagem Natural (PLN) para auferir a intenção do usuário.

Como trabalhos futuros propõe-se a descoberta automática de dispositivos, de forma a automatizar o processo de instalação de um dispositivo inteligente à rede. Possibilidades que podem ser exploradas a resolver esse problema: a utilização de um dos protocolos a seguir por parte do dispositivo: (1) Universal Plug and Play (UPnP), (2) Multicast DNS (mDNS) ou Zero-configuration Networking (zeroconf).

\section{Referências}

Balta-Ozkan, N., Boteler, B. and Amerighi, O. (2014). European smart home market development: Public views on technical and economic aspects across the United Kingdom, Germany and Italy, Energy Research $\&$ Social Science 3: 65-77.

CMUSphinx (2018). Cmusphinx - open source speech recognition toolkit, https://cmusphinx.github.io/.

Coulouris, G. F. (2005). Distributed systems: concepts and design, Pearson Education.

Hansen, M. (2018). Rhasspy voice assistant toolkit, https://github.com/synesthesiam/rhasspy.

HomeAssistant (2018). Home assistant dev docs 0.81.0, https://www.home-assistant.io/docs.

Mahmoud, M. K. (2014). Smart home hub. US Patent 8,724,639.

Moore, R. K. (2003). A comparison of the data requirements of automatic speech recognition systems and human listeners, Eighth European Conference on Speech Communication and Technology.

OASIS (2014). Mqtt oasis standard specifications, http://docs.oasis-open.org/ mqtt/mqtt/v3.1.1/os/mqtt-v3.1.1-os.pdf.

Organization, W. H. (2015). World report on ageing and health, World Health Org.

Portet, Vacher, G. R. M. (2011). Design and evaluation of a smart home voice interface for the elderly: acceptability and objection aspects, Personal and Ubiquitous Computing .

Sethi, P. and Sarangi, S. R. (2017). Internet of Things: Architectures, protocols, and applications, Journal of Electrical and Computer Engineering .

Trappeniers, M. A. F. . F. K. . M. B. . L. (2013). The Internet of Things: The Next Technological Revolution, Computer pp. 24-25.

W3C (2018). Jspeech grammar format, https://www.w3.org/TR/jsgf/. 